\title{
Electron Injection by Dephasing Electrons with Laser Fields
}

\author{
E. Dodd, J. K. Kim and D. Umstadter \\ Center for Ultrafast Optical Science \\ University of Michigan, Ann Arbor, MI 48109
}

\begin{abstract}
The authors seek to review injection concepts for plasma based acceleration. It is shown that regardless of injection mechanism. resultant beams will be similar due to wave structure. Also, most schemes employ the same basic processes, namely the dephasing of electrons by laser fields, and can thus be analyzed with similar approaches.
\end{abstract}

\section{INTRODUCTION}

Lascr-plasma based acceleration coupled with CPA laser technology has become the topic of much current interest in recent years. As a part of this discussion, the question arose as to how to generate and inject fomtosecond-duration electron bunches for wake-field acceleration. Electron beam quality suitable for x-ray generation or high-energy physics has yet to be demonstrated by use of laser wake-fields injected with either RF injectors or wavebreaking. A solution to this problem was recently proposed, [1] in which a second laser pulse, split from the same laser system. is used to inject an clectron bunch into the wake-field. This would have several important advantages, including femtosecond-timescale synchronization and pulse durations, as well as greater simplicity. Subsequently, a number of different papers have proposed variations of this idea $[2,3]$ or further analyzed this concept $\left[\begin{array}{ll}4 & 6\end{array}\right]$.

The concept of laser induced plasma waves to accelerate charged particles is itsclf nearly twenty years old [7]. Wake-field accelerators seek to take advantage of ultra-high acceleration gradients $(>10 \mathrm{GeV} / \mathrm{m}$ ) for electrons based on laserdriven plasma waves [8-10]. possible due to the invention of compact, high-peakpower lasers [11 13]. The plasma-wave electric field gradients are three-orders-ofmagnitude higher than those in conventional RF linacs, because they are not limited by dielectric breakdown. In fact recently, gradients on the order of $1 \mathrm{GeV} / \mathrm{cm}$ have been demonstrated experimentally [14], and accelerate electron beams with transverse emittances that rival current electron guns. However. the plasma wave length is much shorter than that of the RF linac, hence the need for very short pulse injectors. 
Electrons normally oscillate in the plasma wave and cannot be accelerated by the wake-ficld since they are out of phase with it, as in Fig. 1. Electrons that are not part of the plasma wave can become trapped. or continuously accelerated by

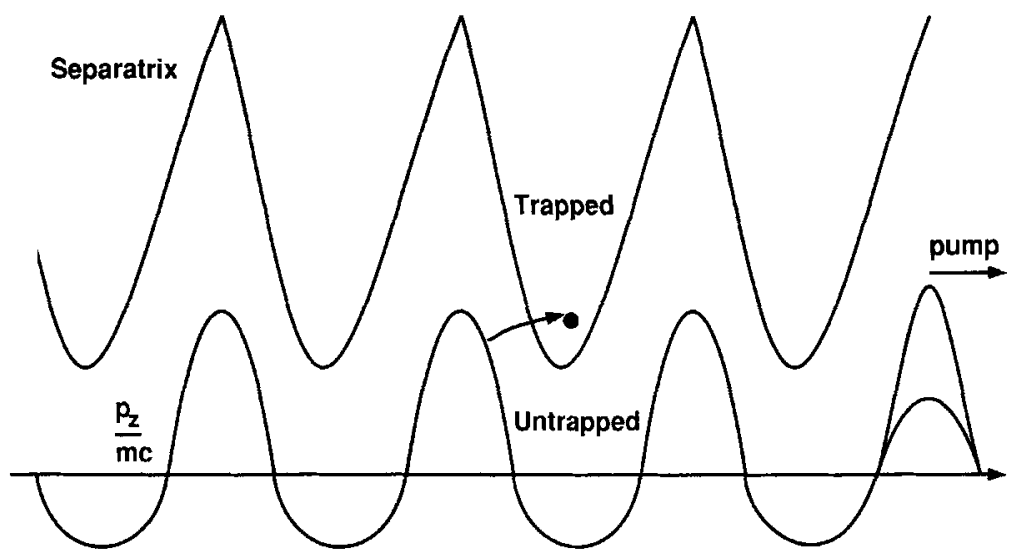

FIGURE 1. Basic profile for LWFA. The pump pulse creates a plasma wave to accelerate electrons. To be accelerated, electrons must cross inside the separatrix.

the wave, provided that they are moving in the correct phase at nearly the phase velocity of the wave [15]. Since this velocity is close to the speed of light, it was gencrally thought that such pre-acceleration can only be accomplished by cxternal injection, such as with a conventional linac. However, the low-field gradient $(<10$ $\mathrm{MeV} / \mathrm{m})[16]$ of a first-stage conventional linac prolongs the time during which beam emittance can grow before the beam becomes relativistic; after this point. self-generated magnetic fields can balance the effects of space charge.

\section{METHODS OF INJECTION}

The LILAC, or Laser Injected Laser ACcelerator, was proposed in [1] to solve the injector problems. It consists mainly of three different stages. First a large amplitude wake-field is generated; second, electrons are dephased; and then thirdly, electrons are trapped in the pump's wake-field due to the dephasing and accelerated. By understanding each of the three parts involved, a description of the injection process can be derived. However, the first part is beyond the scope of this paper. and without loss of generality, no discussion of generation mechanisms will be included. The process of dephasing is quite general, using secondary laser pulses or other methods to move electrons oscillating in the wave across the separatrix for acceleration. These methods can include both the ponderomotive force or the direct field of the laser pulse acting on electrons or through collective effects of the plasma such as waves. Regardless, all methods seek to produce similar results after the injection process has ended. 
The first scheme proposed, [1], used the ponderomotive force of the second, or injection pulse, with an orthogonal geomctry to dephase electrons for trapping, Fig. 2 a). Besides orthogonal, other orientations of the laser pulses are also possible, collinear or counter-propagating. The next variation was the collinear LILAC shown in Fig. 2 b), where the ponderomotive force drives the wave to breaking for
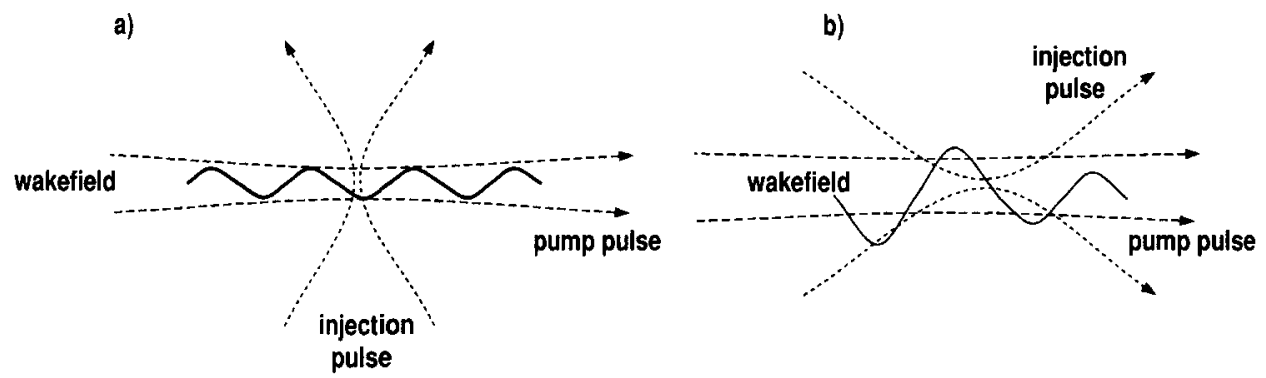

FIGURE 2. a) Schematic of the transverse LILAC accelerator concept. b) Schematic diagram of the collinear LILAC. Ploase note that only the contours of intensity are shown.

injection. Another scheme, [3], again uses the ponderomotive force, but now with two counter-propagating pulses. When overlapped, the beating produces the dephasing for injection, which only occurs when the pulses are overlapped. This way injection is gated on and off for only a short period of time.

Another way a laser pulse may inject electrons is through collective interactions with the plasma. The first example is through the use of a plasma instability, or the so-called self modulated wake lield. This is based on the plasma wave generated from the Raman instability, and forward scattering accelerating the electrons. for example see [14]. It however produces electrons in a continulum and is not of interest. An injection pulse may also produce a wake. The interaction between the wakes of the various pulses will dephase electrons enough for trapping and was studied in [5]. To do this the length of the injection pulse was shorter and more highly resonant than in previous studies, [6], but still used the orthogonal orientation.

The ponderomotive force is really an average of the particle's motion over many optical cycles, as such the dephasing is not directly due to the electric field in the laser pulse. Since field amplitude of the pulse is larger than the ponderomotive force the use of a sub cycle pulse was proposed in [2]. This method produces a short bunch with minimal energy spread, needed for injection into plasma waves.

Besides combining laser pulse characteristics, ionization or density gradients are other possible means to produce dephased electrons. Another effect looked at briefly was trapping due to edge effects. A sharp boundary in the simulation caused the plasma frequency to change abruptly, going from zero in vacuum to full density in a few microns. Oscillating particles will see two frequencies as they move into the vacuum and return, causing them to be dephased, and possibly trapped. This was studied previously by two other groups [17]. Since this sharp boundary does 
not exist in the experiment, we chose to use the solution of G. Bonnaud et. al. to remove this problem from the code, and moved the particle boundary accordingly. Physical boundaries this sharp are difficult to achieve experimentally, but if some method were found it could serve as an efficient injection mechanism.

\section{BUNCH CHARACTERISTICS}

Electrons bunches that have been accelerated have a number of important characteristics. These depend on the plasma wave as much as they depend on the injection process. For example, the first characteristic of interest is simply the number of clectrons in the bunch which is limited by beam loading [18], the limit where the space charge of the bunch effectively screens out the accelerating gradient. This is independent of injection method since it depends only on the wave amplitude and plasma density. For plasma densities of $10^{19} \mathrm{~cm}^{-3}$ and wave amplitude of $1 \mathrm{GeV} / \mathrm{cm}$ this is $n_{b}=\tau \times 10^{8}$ electrons, any method of interest should be capable of injection this many electrons into the wave.

Also important are the energy spread of the bunch and the transverse cmittance. Due to the short plasma wave length the accelerating gradient changes from zero field to more than $1 \mathrm{GeV} / \mathrm{cm}$ over only a few microns, allowing for large changes in electron energy even in short bunch lengths. To eliminate this problem electrons must be injected only over a small phase of the wave, hence the need for gating. The orthogonal laser pulse orientation also solves this problem due to short transit time of the injection pulse across the plasma wave. Wave breaking and longitudinal orientations can inject over large plase ranges giving rise to energy spreads close to $100 \%$. This is much more dependent on injection method than bunch number.

As a bunch leaves the aceolerator it begins to diverge which is characterized by the transverse emittance. Like a conventional accelerating structure, a plasma wave has lirrit on the largest emittance it can hold, or acceptance. This limit can be calculated from the spot size and the strength of the radial electric fields in the wave, an example of which appears in [5]. This may be used as a prediction of emittance produced in plasma wave acceleration. One finds that this value is on the same order as that of more conventional RF photo-cathode guns, however in [14] a value of $0.2 \pi \mathrm{mm} \cdot \mathrm{mrad}$ for normalized transverse emittance was measured. These electrons were produced through wave breaking and hence have $100 \%$ energy spread in contrast to the low emittance. However this docs demonstrate experimentally that with the right injection mechanism very low emittances are possible with laser-plasma based acceleration.

\section{CONCLUSION}

In conclusion we have shown that all injection methods produce similar results, depending on the plasma wave as much as injection process. Additionally most injection schemes are variations on a few basic ideas, either directly dephasing electrons or using collective plasma effects. As yet no best method for injection to 
create an optimal electron bunch for acceleration in plasma waves has been found. which will only be resolved through experiment since all present work has been based on theoretical and numeric work.

We would like to acknowledge the support of the NSF and to thank Torsten Neubert and Gerard Mourou for many valuable discussions. Computing services were provided by the University of Michigan Center for Parallel Computing, which is partially funded by NSF grant CDA-92-14296.

\section{REFERENCES}

1. D. Umstadter, J. K. Kim and E. Dodd. Phys. Rev. Lett. 76, 2073 (1996).

2. B. Rau, T. Tajima, and H. Hojo, Phys. Rev. Lett. 78, 3310 (1997).

3. E. Esarey, et.al., Phys. Rev. Lett. 79, 2682 (1997).

4. J. L. Bobin, Advanced Accelerator Concepts, AIP Conf. Proc. 398, S. Chattopadhyay, ed., (1997).

5. R. G. Hemker, et.al., Phys. Rcv. E.57, 5920 (1998).

6. J. K. Kim, E. Dodd and D. Umstadter. submitted to Phys. Rev. E.

7. T. Tajima and J. M. Dawson, Phys. Rev. Lett. 43, 267 (1979).

8. P. Sprangle et al.. Appl. Phys. Lett. 53, 2146 (1988).

9. L. M. Gorbunov and V. I. Kirsanov, Sov. Phys. JETP 66, 290 (1987): H. Hamster et al., Phys. Rev. Lett. 71, 2725 (1993); K. Nakajima et al., Phys. Rev. Lett. 74, 4428 (1995).

10. See e.g., Admanced Accelerator Concepts, Fontana, WI, 1994, Amer. Inst. of Conf. Proc: No. 335, P. Schoessow, ed., (AIP Press, New York, 1995) and references cited therein.

11. P. Maine et al., IEEE J. Quantum. Electron. 24, 398 (1988).

12. G. Mourou and D. Unstadter, Phys. Fluids B 4, 2315 (1992).

13. M. D. Perry and G. Mourou, Science 264, 917 (1994).

14. R. Wagner et al., Phys. Rev. Lett. 78, 3125 (1997).

15. E. Esarey and M. Pilloff, Phys. Plasmas 21432 (1995): T. Katsouleas et al.. in Advanced Accelerator Concepts. AIP Conf. Proc. 130 (1985).

16. S. Humphries Jr., Principles of Charged Particle Accelerators (Wiley, New York, 1986).

17. G. Bonnaud, D. Teychennc, and J. L. Bobin. Europhys. Lett. 26, 91 (1994); S. V. Bulanov et al., Sov. J. Plasma Phys. 16, 444 (1990).

18. T. Katsouleas et al., Particle Accelerators, 2281 (1987). 